\title{
An Objective Diagnosis Model with Integrated Metabolic and Immunity Parameters for Phlegm-Dampness Constitution
}

\author{
Yanchun Huang, ${ }^{1}$ Shanshan Guo, ${ }^{2}$ Jun Yang, ${ }^{1}$ Yangfan Tang, ${ }^{2}$ Xinghua Zhu, \\ and Sichong Ren $\mathbb{1}^{2,3}$ \\ ${ }^{1}$ Department of Laboratory Medicine, The First People's Hospital of Longquanyi District, Chengdu, \\ West China Longquan Hospital Sichuan University, Chengdu 610100, China \\ ${ }^{2}$ Center for Translational Medicine, Sichuan Academy of Traditional Chinese Medicine, \\ Translational Chinese Medicine Key Laboratory of Sichuan Province, Chengdu 610041, China \\ ${ }^{3}$ Department of Nephrology, Clinical Medical College and the First Affiliated Hospital of Chengdu Medical College, \\ Chengdu, China
}

Correspondence should be addressed to Sichong Ren; sichongren@yahoo.com

Received 24 July 2021; Revised 6 December 2021; Accepted 8 January 2022; Published 4 February 2022

Academic Editor: Xue-Rui Wang

Copyright (c) 2022 Yanchun Huang et al. This is an open access article distributed under the Creative Commons Attribution License, which permits unrestricted use, distribution, and reproduction in any medium, provided the original work is properly cited.

\begin{abstract}
Background. According to Chinese constitutional theory, people can be divided into nine constitutions, which represent distinctive vulnerability to different diseases such as metabolic syndrome, atherosclerosis, and immunity-related disease, and so forth in modern medicine, phlegm-dampness constitution (PDC) is one of the nine constitutions, which is susceptible to metabolic syndrome (MS) and atherosclerosis that associate with lipid metabolism and immunity dysregulation closely. Objectives. In this study, we aimed to investigate the metabolic and immunity profiles of phlegm-damp constitution (PDC), including metabolites, lymphocytes distribution, and inflammatory cytokines. Methods. A total of 74 patients with PDC and 66 individuals with gentle constitution (GC) were enrolled in this study. We utilized biochemical methods to detect metabolic parameters, flow cytometry to survey T/B/NK/NKT lymphocyte subgroups distribution, and ELISA to assay inflammatory cytokines. Results. The subjects with PDC had higher GLU, AI TC, TG, and LDL-C and lower HDL-C levels. The immunity profile indicated that PDC subjects had higher percentage of WBCs, neutrophils, lymphocytes, B cells, and natural killer T cells compared with subjects with GC $(P<0.05)$. Serum levels of IL-10 decreased significantly in the subjects with phlegm-damp constitution, whereas IL-12 levels increased dramatically in the PDC group compared with the GC group (both $P<0.05$ ). Additionally, logistic regression identified four independent variables (GLU, TG, LDL-C, and lymphocytes) that were highly correlated with PDC $(P<0.05)$. The area under the curve of the receiver operating characteristic curve was 0.878 , which indicated the data were reliable to distinguish the subjects with PDC from the ones with GC. Conclusion. Phlegm-damp constitution was prone to hyperglycemia and hyperlipidemia syndrome, promoting the occurrence and progression of metabolic-related diseases. Interestingly, proinflammatory cells and cytokines were activated in the PDC group as well. Our findings could offer a profile of early screening indicators to identify highrisk patients of metabolic- and immunity-related diseases from Chinese constitution.
\end{abstract}

\section{Introduction}

Chinese constitutional theory was proposed in the 1970s and has been widely used in clinical practice by traditional Chinese medical (TCM) doctors for disease management and health promotion [1]. According to this theory, people are divided into nine constitutions, which comprise one balanced constitution (normality) and eight imbalanced constitutions (phlegm-dampness, damp-heat, yang-deficient, blood stasis, yin-deficient, qi-deficient, qi-stagnation, and special intrinsic constitution) based on the Classification and Determination of Constitution [2]. Constitution is an individual life-related stable phenotype, which is considered as an integrated indicator of physical and psychological 
characteristics individually affected by hereditary and acquired environment together. A plenty of studies from TCM has showed that it tendentiously determined an individual's susceptibility to certain diseases, and it was regarded as a risk factor of its related diseases. Eventually, these imbalanced constitutions could initiate and accelerate different diseases [3].

Phlegm-dampness constitution (PDC) is one of the eight imbalanced constitutions and most common type in TCM studies. The following are the common characteristics of PDC: fat, greasy coating on the tongue, slippery pulse, copious and sticky sweat, dark yellow facial complexion, face and eyelid edema, copious phlegm, drowsiness, heavy limbs, etc. [3]. People with PDC are unable to adapt to the moist environment and vulnerable to dampness syndrome. Studies demonstrated that PDC patients are susceptible to diabetes, metabolic syndrome (MS), and cardiovascular disease (CVD) [4, 5]. MS is termed as a group of interrelated cardiometabolic risk factors, including elevated fasting glucose, elevated triglycerides (TGs), reduced high-density lipoprotein (HDL), elevated blood pressure, and central obesity [6]. Approximately $30 \%$ of adults had MS worldwide, and the prevalence of the disease was increased in various countries and regions recently [7, 8]. Studies demonstrated that MS contribute to CVD in patients remarkably $[9,10]$. In TCM theory, PDC is considered as the early stage of MS. Therefore, diagnosis and intervention of PDC are important to decrease the incidence of MS and CVD.

Immunity plays a critical role in the occurrence and development of MS, diabetes, and CVD [11-13]. TCM suggested that dysfunction of body's spleen and stomach could affect the metabolism and immunity balance, which gradually become PDC [14]. Similarly, modern medicine confirmed that the spleen is a vital organ of immune cell development and immune function regulation [15]. Hitherto, there is no report about immunological profile including immune cell subtypes and cytokine parameters in PDC subjects.

Thus, this study investigated lipid metabolites, lymphocyte subtypes distribution, and inflammatory cytokines in PDC. Results had indicated that lipid metabolism was dysregulated and accompanied with a higher percentage of $\mathrm{B}$ cells and natural killer T cells in PDC patients. It offered us a point of connecting PDC with CVD in these two medicine theories, meanwhile, which provided novel ideas for objective screen and intervention of PDC in future.

\section{Methods}

2.1. Patients. All subjects aged 35-84 years were selected from the outpatients of the Cardiology Department and Health Management Center, West China Longquan Hospital Sichuan University, from January 2019 to June 2020. The subjects were judged independently by two experienced doctors who calculated scores according to the criteria evaluation of Chinese constitution [3]; for example, whitegreasy tongue coating (5 scores) and swollen tongue proper (4 scores) with slippery pulse (4 scores). The phlegm- dampness-related symptoms include the sensation of tiredness, listlessness, and sick feeling (3 scores), slightly edematous eyelids (2 scores), chest stuffiness (3 scores), sleepiness (2 scores), fatty and soft abdomen (2 scores), abundant mucous or thin white sputum (1 score), swollen lower limbs (2 scores), pale-yellow complexion (1 score), and slimy sensation in the mouth (1 score). Individuals with total scores $>9$ were judged as PDC. However, gentle constitution (GC) showed some symptoms, such as calm, strong pulse, and pink tongue with thin white coating, with total score $<9$ according to Chinese constitution criteria. In this study, the inclusion criteria of PDC are outlined as follows: (a) transformed scores of the phlegm-dampness-related symptoms are greater than 40; and (b) two doctors have no controversy. The inclusion criteria of GC are outlined as follows: (a) transformed scores of are greater than 60 and transformed scores of imbalanced constitutions are less than 30; and (b) two doctors have no controversy. Subjects were excluded if they exhibited any of the following: (a) two doctors have a controversy; (b) subjects with other autoimmune diseases, cancer, systemic diseases, and other serious diseases; or (c) subjects in the acute phase of the disease (for example, infection).

2.2. Main Reagents and Equipment. The main reagents and equipment used in this study were as follows: automatic blood cell counter BC6900 and supporting reagents (Mindray, China); automatic biochemical apparatus type AU5800 (Beckman Coulter, USA), and biochemical reagent (Orienter, China); flow cytometry DxFLEX6 and antibodies for lymphocytes identification (Beckman Coulter Life Sciences); ELISA for cytokine testing Kits (R\&D).

2.3. Leukocyte Count. Blood from subjects was collected in a tube with EDTA (anticoagulant) and assessed using the automatic blood cell counter BC6900, according to the standard operating procedure (SOP).

2.4. Biochemical Measurement. Whole blood from subjects was collected, and their serum was isolated. Fasting blood glucose (GLU), total cholesterol (TC), triglyceride (TG), low-density lipoprotein-cholesterol (LDL-C), high-density lipoprotein-cholesterol (HDL-C), apoprotein A (apoA), and apoprotein $\mathrm{B}$ (apoB) were measured by the colorimetric method on an automatic biochemical apparatus type AU5800 (Beckman Coulter), according to its SOP. The arteriosclerosis index (AI) was calculated using the formula (TC - HDL)/HDL.

2.5. Flow Cytometry Analysis. Peripheral blood samples were obtained from subjects. For antibody staining, $10 \mathrm{~mL}$ of the indicated antibody was mixed gently to $100 \mathrm{~mL}$ blood sample and incubated at room temperature in the dark for $15 \mathrm{~min}$. Subsequently, $500 \mathrm{~mL}$ hemolysin buffer was added to the same sample tube and mixed gently and incubated at room temperature in the dark for an additional $15 \mathrm{~min}$, followed by an addition of $500 \mathrm{~mL}$ sheath buffer for 
detection. The indicator antibodies were as follows: CD45FITC/CD4-RD1/CD8-ECD/CD3-PC5 was used for T/ B lymphocyte subsets (6607013, Beckman Coulter Life Sciences), and CD45-FITC/CD3-RD1/CD19-ECD/CD56PC5 was used for B/natural killer (NK)/NKT lymphocyte subset (6607073, Beckman Coulter Life Sciences). The distribution of all the circulating T, B, NK, and NKT cell subsets was assessed by flow cytometry (DxFLEX6; Beckman Coulter Life Sciences) according to the manufacturer's protocol. The threshold for positive staining was determined using fluorescence minus one (FMO) control. Additionally, the performance of the instrument was verified daily using the tracking system to ensure the consistency of flow cytometry.

2.6. Cytokines Measurement. Blood samples were collected from subjects, and levels of IL-4, IL-2, IFN-g, IL-6, IL-10, and IL-12 were detected using commercial ELISA kits (R\&D), according to the manufacturer's instructions.

2.7. Statistical Analysis. Statistical analysis was performed using SPSS Statistics 22.0. for Windows (SPSS, Inc., Chicago, IL, USA). All figures were constructed using Prism software (GraphPad Prism 7). The values were expressed as mean \pm SD or median $\left(25^{\text {th }}-75^{\text {th }}\right.$ percentile). For parametric data, we used the independent samples $t$-test, while nonparametric data were evaluated using the Mann-Whitney $U$ test. Pearson's correlation coefficient was used to assess correlations. Univariate logistic regression analysis was performed for all indexes associated with PDC. The identified variables for which the $P$-value $<0.05$ in the univariate analysis were further assessed with binary logistic regression (stepwise forward), and the probability of having PDC was obtained. Then, probability estimates were used to plot the receiver operating characteristic (ROC) curve. We used the Hosmer-Lemeshow goodness-of-fit test to assess model fit. The equation of this model is $P(y=1)=$ $e^{y} /\left(1+e^{y}\right), \quad y=\beta 0+\beta 1 X 1+\beta 1 X 1+\beta 2 X 2+\beta 3 X 3+\ldots+\beta n X n$, $e=2.718$. For all the tests, the statistical significance was set at $P<0.05$.

\section{Results}

3.1. Metabolites and Routine Blood Testing Parameters of Recruited Subjects. Among recruited subjects, 74 patients had PDC and 66 volunteers were controls (gentle constitution). The cohort comprised $56.8 \%$ and $51.5 \%$ females in PDC and GC groups, respectively. As shown in Table 1, the group of PDC had higher diabetes prevalence and cardiovascular disease prevalence than the group of gentle constitution. The median age was $51.20 \pm 11.80$ years and $49.41 \pm 11.55$ years in PDC and GC groups, respectively. The two groups were similar in terms of age and gender. The PDC group had higher levels of GLU, AI, TC, TG, LDL-C, WBC, lymphocyte counts, and neutrophil counts from routine blood testing but a lower HDL-C level in lipid metabolism (Table 1).
3.2. Lymphocytes Subpopulations Distribution. Flow cytometry was applied to investigate the distribution of leukocyte subpopulations in the two groups. Compared with the GC group, the PDC group had higher events of lymphocytes, B cells, and NKT cells $(P<0.05)$. Percentages of $\mathrm{B}$ and NKT cells were higher in the PDC group than GC group. However, the GC group had a higher percentage of total lymphocytes than the PDC group. Besides, percentages of $\mathrm{T}$ and NK cells were similar in both groups (Table 2). These cytometry photographs of lymphocytes distribution displayed lymphocyte subsets, NKT and NK cells, and effector NK $\left(\mathrm{CD} 16^{+} \mathrm{CD} 56^{\text {+high }}\right)$ representing immunity states of the two groups (Figure 1).

3.3. Serum Cytokine Levels. Serum IL-4, IL-2, IL-6, IL10, IL12 , and IFN- $\gamma$ cytokine levels reflect inflammatory states; we investigated these inflammatory cytokines in PDC and GC groups (Figure 2). In this study, the serum levels of antiinflammatory cytokine IL-10 decreased significantly in the PDC group compared with the GC group $(P=0.015)$. However, the proinflammatory cytokine IL-12 level increased dramatically in the PDC group compared with GC group $(P=0.038)$. Other cytokines IFN- $\gamma$, IL-4, IL-2, and IL-6 showed no difference between the PDC and GC groups.

3.4. Logistic Regression Model for PDC. Logistic regression analyses (stepwise, forward) were used to establish three identifiable PDC models. There were four independent variables in the combined model: GLU (odds ratio (OR): 1.470, 95\% CI: 1.069-2.021), TG (OR: 1.759, 95\% CI: 0.897-3.450), LYM (OR: 0.858, 95\% CI: 0.773-0.952), and LDL-C (OR: 2.512, 95\% CI: 1.193-5.289) (Table 3). GLU (OR: 1.470, 95\%CI: 1.154-1.873), TG (OR: 2.138, 95\%CI: 1.370-3.337), and LDL-C (OR: 2.295, 95\%CI: 1.300-4.054) were included as covariates in the metabolic model (Table 3). In the immunity model, the number of B cells (OR: 0.993, 95\% CI: 0.988-0.999) and IL-10 (OR: 0.999, 95\%CI: 0.998-1.000) were include (Table 3). The Hosmer-Lemeshow goodness-of-fit test was also implemented, which indicated that there was no overfitting of the models. Besides, we drew the receiver operating characteristic curve to check sensitivity and specificity parameters. The results showed that the area under the curve of the metabolic model, the immunity model, and the combined model were 0.868 , 0.825 , and 0.878 , respectively (Figure 3 ). The sensitivity and specificity of the combined model were 0.727 and 0.977 , respectively, when the cutoff was 0.610 (Table 4 ).

\section{Discussion}

In this study, we found that the PDC group had higher glucose, TG, TC, and LDL-C levels compared with the gentle constitution group. Some investigators found that imbalanced constitutions, especially PDC, had a close correlation with abnormal glucose and dyslipidemia [16-18]. In addition, researchers have explained these phenotypes from the molecular level that five genes and six SNPs were identified differently between PDC and GC. These genes and SNPs 
TABLE 1: Metabolites routine blood testing parameters of subjects

\begin{tabular}{|c|c|c|c|}
\hline Parameters & $\operatorname{PDC}(n=74)$ & $\mathrm{GC}(n=66)$ & $P$ \\
\hline Sex(male/female) & $32 / 42$ & $32 / 34$ & 0.534 \\
\hline Age, years & $51.20 \pm 11.80$ & $49.41 \pm 11.55$ & 0.265 \\
\hline Diabetes (\%) & $31(41.89 \%)$ & $7(10.61 \%)$ & $<0.001$ \\
\hline Receiving glyburide monotherapy (\%) & $4(5.41 \%)$ & $1(1.54 \%)$ & 0.221 \\
\hline History of coronary artery disease & $10(15.63 \%)$ & $1(1.54 \%)$ & 0.008 \\
\hline Receiving atorvastatin monotherapy (\%) & $1(1.35 \%)$ & $0(0.00 \%)$ & 0.529 \\
\hline $\mathrm{GLU}, \mathrm{mmol} / \mathrm{L}$ & $7.73(5.01-12.81)$ & $5.26 \pm 1.29$ & $<0.001$ \\
\hline AI & $5.17(2.56-4.48)$ & $2.33(1.90-2.83)$ & 0.024 \\
\hline $\mathrm{TC}, \mathrm{mmol} / \mathrm{L}$ & $5.87(4.81-6.52)$ & $4.63 \pm 0.96$ & 0.001 \\
\hline $\mathrm{TG}, \mathrm{mmol} / \mathrm{L}$ & $3.81(1.84-3.81)$ & $1.65 \pm 0.80$ & $<0.001$ \\
\hline $\mathrm{HDL}-\mathrm{C}, \mathrm{mmol} / \mathrm{L}$ & $1.27 \pm 0.43$ & $1.44 \pm 0.36$ & 0.012 \\
\hline $\mathrm{LDL}-\mathrm{C}, \mathrm{mmol} / \mathrm{L}$ & $3.75(2.93-4.17)$ & $2.77 \pm 0.64$ & 0.001 \\
\hline apoA, g/L & $1.16 \pm 0.27$ & $1.25 \pm 0.25$ & 0.093 \\
\hline apoB, g/L & $1.12 \pm 0.34$ & $1.07 \pm 1.01$ & 0.753 \\
\hline WBC, $\times 10^{9} / \mathrm{L}$ & $7.29(5.54-7.54)$ & $6.24(4.86-7.54)$ & 0.002 \\
\hline $\mathrm{N}, \times 10^{9} / \mathrm{L}$ & $4.80(3.37-5.44)$ & $3.55(2.78-4.99)$ & $<0.001$ \\
\hline $\mathrm{LYM}, \times 10^{9} / \mathrm{L}$ & $1.98(1.47-2.51)$ & $1.78 \pm 0.76$ & 0.028 \\
\hline NLR & $2.37(1.88-3.08)$ & $1.94(1.25-2.24)$ & 0.302 \\
\hline $\mathrm{PLT}, \times 10^{9} / \mathrm{L}$ & $182.20 \pm 56.30$ & $184.26(166.00-216.50)$ & 0.832 \\
\hline PLR & $125.59(65.50-140.50)$ & $98.7(74.00-135.00)$ & 0.298 \\
\hline $\mathrm{RBC}, \times 1012 / \mathrm{L}$ & $6.41 \pm 16.33$ & $4.60 \pm 0.65$ & 0.374 \\
\hline HCT & $42.03(38.2-47.35)$ & $41.91 \pm 5.19$ & 0.912 \\
\hline $\mathrm{HB}, \mathrm{g} / \mathrm{L}$ & $137.23 \pm 18.99$ & $140.12 \pm 15.77$ & 0.339 \\
\hline
\end{tabular}

PDC: phlegm-damp constitution, GC: gentle constitution, GLU: fasting blood glucose, AI: arteriosclerosis index, TC: total cholesterol, TG: triglyceride, HDLC: high-density lipoprotein-cholesterol, LDL-C: low-density lipoprotein-cholesterol, apoA: apoprotein A, apoB: apoprotein B; N: neutrophils; LYM: lymphocytes, NLR: the ratio of neutrophil/lymphocyte, PLT: platelets, PLR: the ratio of platelet/lymphocyte, RBC: red blood cell, HCT: hematocrit, HB: hemoglobin. Bold font indicates $P$ value less than 0.05 .

TABLE 2: The levels of mononuclear cells in blood.

\begin{tabular}{lccc}
\hline Variables & PDC $(n=74)$ & GC $(n=66)$ & \multicolumn{1}{c}{$P$} \\
\hline Lymphocytes, \% & $27.81 \pm 3.74$ & $24.23 \pm 9.39$ & $\mathbf{0 . 0 2 1}$ \\
Lymphocytes, events/ $\mu \mathrm{l}$ & $2155.32 \pm 619.99$ & $2096.88 \pm 907.99$ & $\mathbf{0 . 4 2 5}$ \\
B, \% & $9.17 \pm 2.91$ & $7.03 \pm 3.99$ & $\mathbf{0 . 0 0 5}$ \\
B, events $/ \mu \mathrm{l}$ & $198.86 \pm 73.56$ & $142.15 \pm 98.10$ & $\mathbf{0 . 0 0 3}$ \\
T, \% & $71.40 \pm 10.46$ & $68.50 \pm 7.22$ & 0.134 \\
T, events $/ \mu \mathrm{l}$ & $1492.80 \pm 686.28$ & $1499.09 \pm 375.83$ & 0.958 \\
NKT, \% & $6.46 \pm 2.44$ & $4.95 \pm 3.86$ & $\mathbf{0 . 0 3 1}$ \\
NKT, events $/ \mu \mathrm{l}$ & $114.93 \pm 105.00$ & $112.41 \pm 75.55$ & $\mathbf{0 . 0 2 3}$ \\
NK, \% & $17.56 \pm 9.37$ & $19.09 \pm 5.90$ & 0.363 \\
NK, events $/ \mu \mathrm{l}$ & $371.35 \pm 245.71$ & $419.74 \pm 173.68$ & 0.289 \\
\hline
\end{tabular}

PDC: phlegm-damp constitution, GC: gentle constitution, B: B lymphocytes, T: T lymphocytes, NKT: natural killer T (NKT) cells, NK: natural killer cells. \% means percentage of cells. Events $/ \mu$ indicates counting of cell absolutely. $P$ values less than 0.05 represent significant difference.

were involved in gluconeogenesis and thermoregulation, as well as the process of lipid metabolism and brown fat cells differentiation, which indicated that individuals with PDC were susceptible to metabolic disorders from the genetic level $[19,20]$. We found that B cells were significantly higher in the subjects with PDC compared with that of GC. Some scholars suggested that B cells played a central role in the development of insulin resistance, which could make an interpretation of obesity-associated insulin resistance caused by activation of B cells [21]. Many evidence also supported the activation of $\mathrm{B}$ cells was significantly higher in obese patients [22]. Increased B cells in PDC may be accounted for the obese characteristic of PDC dysregulated B cell immunity, which contributed to diabetes mellitus. The existing literature suggests that increased lymphocyte counts are associated with decreased insulin sensitivity and higher risk to develop diabetes [23]. Furthermore, individuals with DM have increased memory CD4+ T cells populations and decreased regulatory $\mathrm{T}$ cells, which create a proinflammatory state $[24,25]$. In conclusion, these data suggest that patients with diabetes may have higher lymphocytes characterized by a functional profile skewed towards a proinflammatory state. In our study, the individuals with PDC had higher lymphocyte counts than the ones with GC, suggesting our results of the present study were consistent with these previous studies.

NKT cells are a nonconventional subtype of CD4 ${ }^{+}$T cells, which firstly described in mice by Bendelac in 1994 [26]. Its cellular surface markers are constituted by NK cell marker $\mathrm{CD}_{56}{ }^{+}$and $\mathrm{T}$ cell receptors together. Usually, it recognizes 

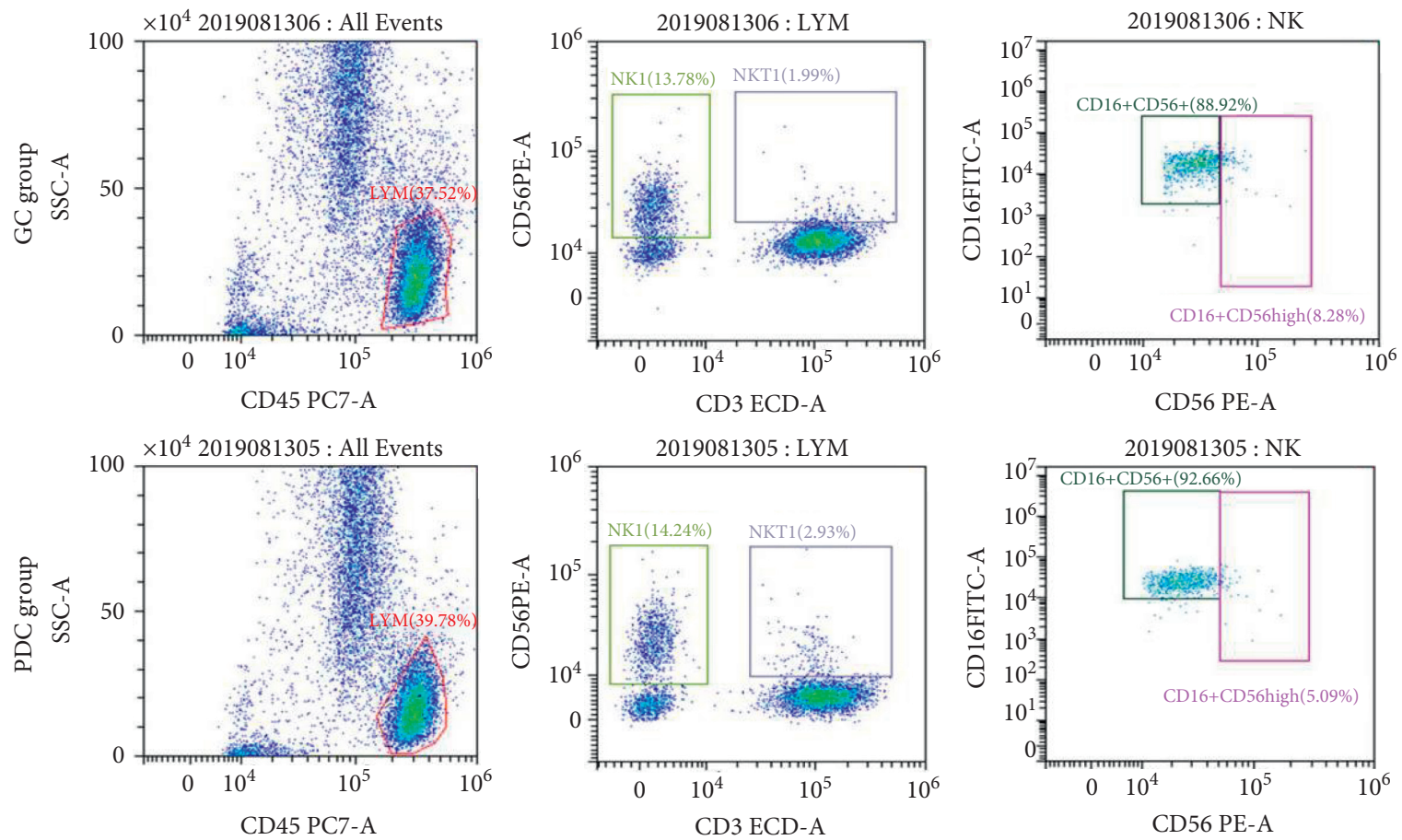

FIGURE 1: Lymphocyte subpopulations distribution in PDC and GC groups. The left lane two photos represent total white cells, and these marked cell clusters by red circles indicate lymphocytes. The medial lane two photos represent NK and NKT cells. The right lane two photos represent $\mathrm{CD} 16^{+} \mathrm{CD} 56^{+}$and $\mathrm{CD} 16^{+} \mathrm{CD} 56^{\text {thigh }}$.

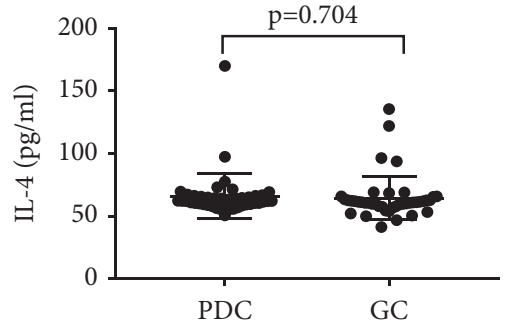

(a)

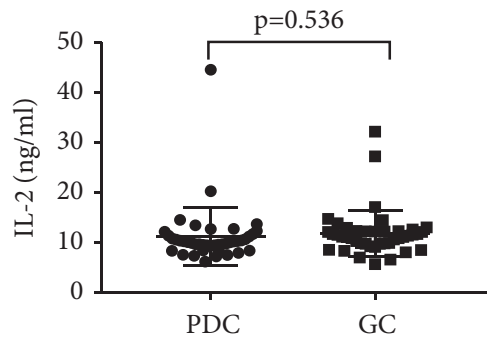

(d)

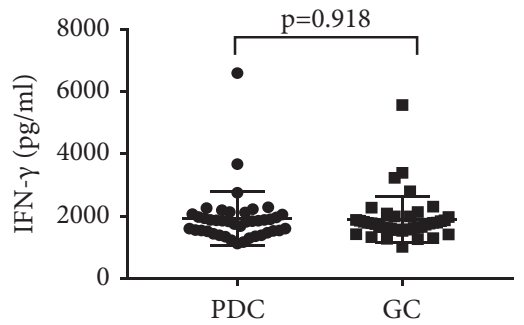

(b)

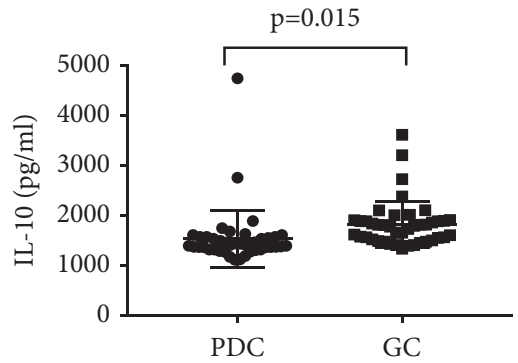

(e)

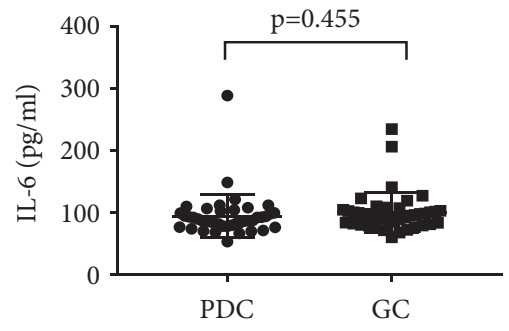

(c)

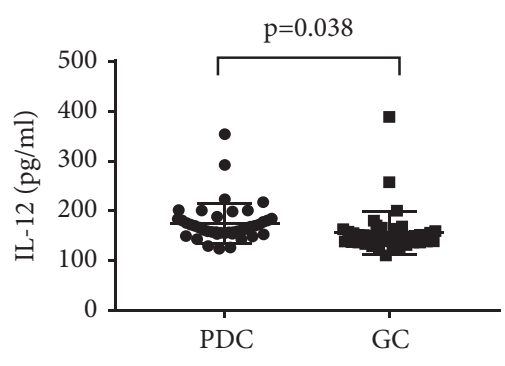

(f)

FIGURE 2: Inflammatory cytokines in the serum of PDC and GC groups. The scatter plot of cytokines for PDC (phlegm-damp constitution) and GC (gentle constitution). IFN- $\gamma$ : interferon $\gamma . P$ values less than 0.05 represent significant difference.

lipid molecules via CD1d but not by major histocompatibility complex (MHC) presenting. Therefore, NKT cells are good executors, which orchestrate innate immune, adaptive immune, and lipid metabolism finely [27]. It has been shown that circulating NKT cells in obese patients were lower than healthy controls [28]. Researchers presumed that a number of NKT cells were stored in adipose tissue [29], so that it could recognize endogenous glycolipids and phospholipids easily to play a critical role in metabolic inflammation modulation [30-32]. Recently, a few studies have suggested that NKT cells played an important role in human atherosclerosis development $[33,34]$. Here, we found NKT cell 
TABLE 3: Variables used in different models.

\begin{tabular}{lcccccc}
\hline Model & Variables & $\beta$ & SE & Wald & $P$ & OR (95\% CI) \\
\hline \multirow{4}{*}{ The metabolic model } & GLU & 0.385 & 0.123 & 9.754 & 0.002 & $1.470(1.154,1.873)$ \\
& TG & 0.76 & 0.227 & 11.193 & 0.001 & $2.138(1.370,3.337)$ \\
& LDL-C & 0.831 & 0.290 & 8.194 & 0.004 & $2.295(1.300,4.054)$ \\
The immunity model & Constant & -6.619 & 1.253 & 27.905 & $<0.001$ & 0.001 \\
& Number of B cells & -0.007 & 0.003 & 5.208 & 0.022 & $0.993(0.988,0.999)$ \\
& IL-10 & -0.001 & 0.001 & 2.684 & 0.101 & $0.999(0.998,1.000)$ \\
& Constant & 2.987 & 1.142 & 6.844 & 0.009 & 19.828 \\
The combined model & LYM & -0.153 & 0.053 & 8.391 & 0.004 & $0.858(0.773,0.952)$ \\
& GLU & 0.385 & 0.162 & 5.63 & 0.018 & $1.470(1.069,2.021)$ \\
& TG & 0.565 & 0.344 & 2.697 & 0.101 & $1.759(0.897,3.450)$ \\
& LDL-C & 0.921 & 0.380 & 5.884 & 0.015 & $2.512(1.193,5.289)$ \\
\hline
\end{tabular}

$\beta$ : regression coefficient, SE: standard error, Wald: Wald chi-square value, CI: confidence interval, OR: odds ratio, GLU: fasting blood glucose, TG: triglyceride, LDL-C: low-density lipoprotein-cholesterol, B: B lymphocytes, LYM: lymphocytes. $P$ values less than 0.05 represent significant difference.

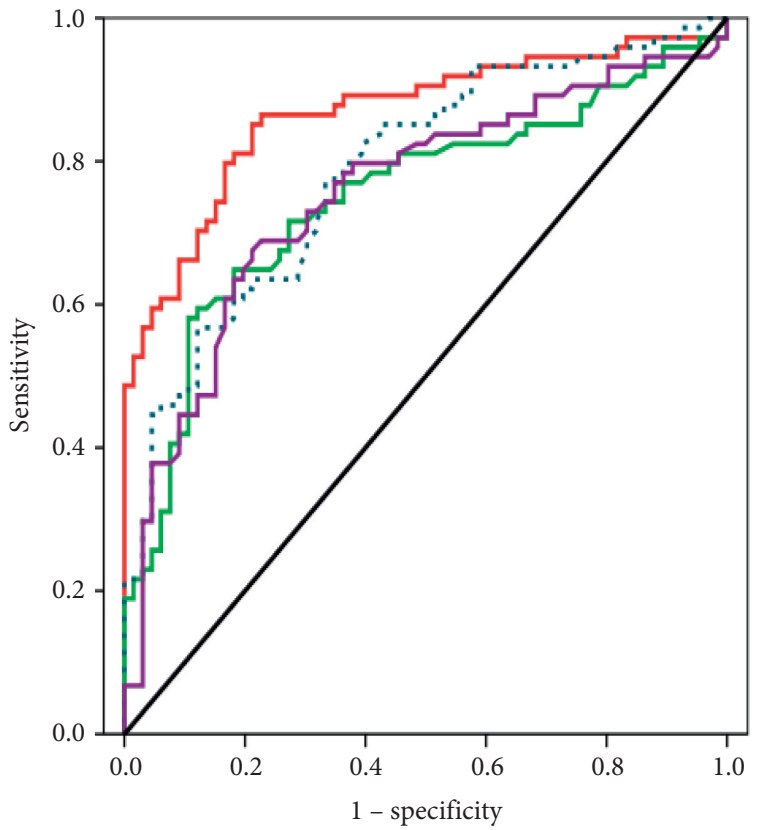

curve designation

— the metabolic model AUC:0.868

— GLU AUC: 0.751

…... TG AUC: 0.787

— LDL-C AUC: 0.755

- the reference

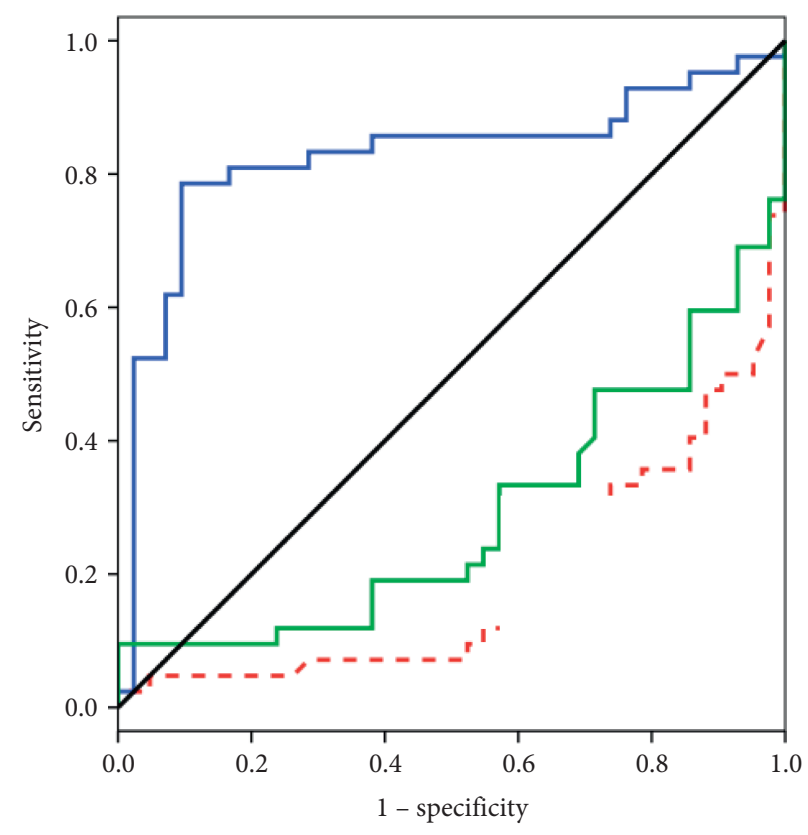

curve designation

- the immunity model AUC $=0.825$

- number of B cells AUC $=0.288$

- - - IL-10 AUC=0.186

— the reference

Figure 3: Continued. 


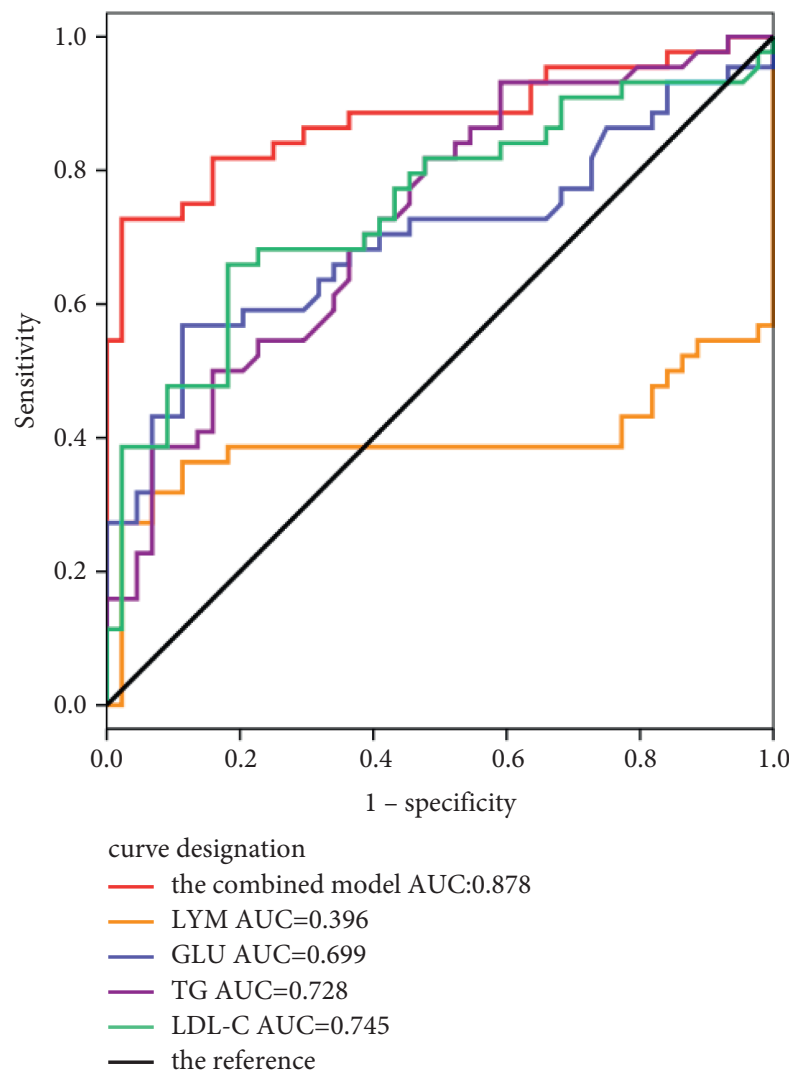

(c)

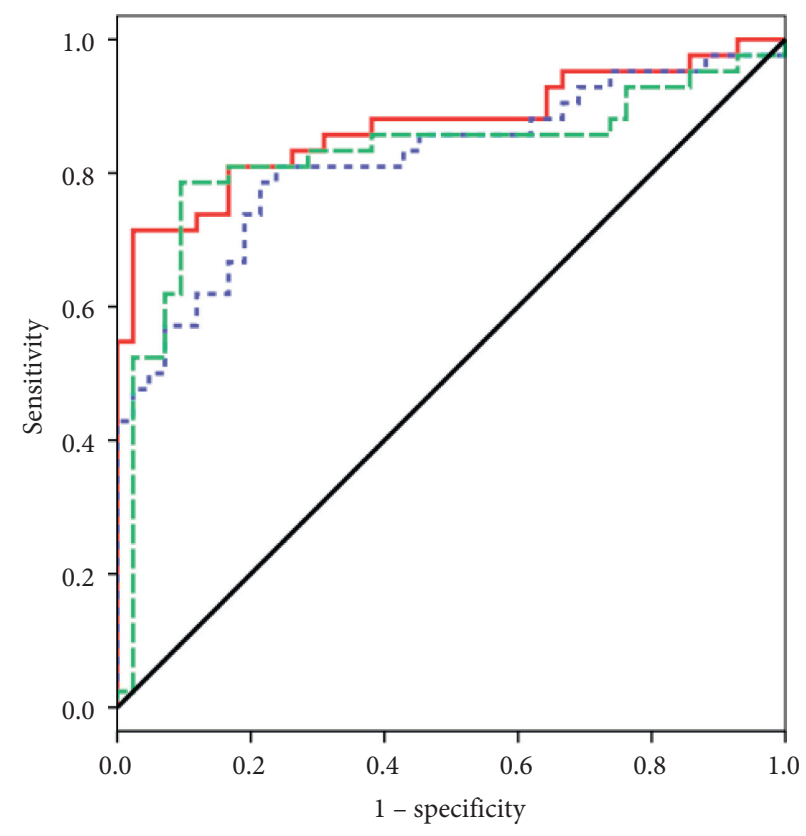

curve designation

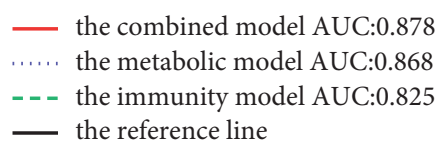

(d)

FIgURE 3: Area under the receiver operating characteristic curve. (a) ROC analysis for the metabolic model and the variables of GLU (fasting blood glucose), TG (triglyceride), and LDL-C (low-density lipoprotein-cholesterol). AUC = area under the ROC curve. (b) ROC analysis for the immunity model and the variables of number of B cells and IL-10. (c) ROC analysis for the combined model and the variables of GLU, TG, LDL-C, and LYM (lymphocyte). (d) ROC analysis for the combined model, the metabolic model, and the immunity model.

TABLE 4: Performance of the models distinguishing subjects with PDC from subjects with GC.

\begin{tabular}{lcccc}
\hline Models $^{1}$ & AUC $(95 \%$ CI $)$ & $P^{2}$ & Sensitivity & Specificity $^{\text {Cutoff }^{3}}$ \\
\hline The metabolic model & $0.868(0.806,0.929)$ & $<0.001$ & 0.851 & 0.788 \\
The immunity model & $0.825(0.727,0.924)$ & $<0.001$ & 0.786 & 0.905 \\
The combined model & $0.878(0.801,0.954)$ & $<0.001$ & 0.727 & 0.563 \\
\hline
\end{tabular}

${ }^{1}$ The models were constructed by logistic regression analyses (stepwise, forward). ${ }^{2} \mathrm{P}$ values for the significance of differences between AUCs and 0.5 by the Wilcoxon rank-sum test. ${ }^{3}$ The optimal cut-point values in ROC (the receiver operating characteristic curve) analyses were defined by the Youden index. PDC: phlegm-damp constitution, GC: gentle constitution, CI: confidence interval, AUC: the area under the curve.

expansion increased in PDC, which may be contributed to diabetes and metabolic syndromes at early stage for initiating metabolic inflammatory cascades in TCM theory. Undeniably, NKT cells play a major role in immunoregulation of PDC, which accounts for that the ones with PDC are especially susceptible to ending with MS and CVD. More detail mechanisms of NKT regulating PDC outcomes need to be elucidated in future.

Chronic inflammation is a crucial culprit for development of diabetes mellitus and cardiovascular diseases $[35,36]$. Inflammation is finely regulated by proinflammatory cytokines and anti-inflammatory cytokines coordinately. In this study, we found that the anti-inflammatory cytokine IL-10 was decreased, whereas proinflammatory IL-12 was increased in the PDC group. Studies demonstrated that IL-10 could improve impaired insulin signaling caused by proinflammatory cytokines [37]. In vivo studies showed that IL-10 could prevent IL-6 or lipid-induced insulin resistance [38]. Moreover, IL-10 was testified to be decreased in MS as well [39]. These previous data support the finding that IL-10 could be a negative biomarker of PDC. Inversely, the proinflammatory IL-12 may be an indicator of PDC and its complications.

Hitherto, PDC is diagnosed mainly according to doctors' consulting experience. So, objective markers and quantitative parameters are needed urgently to offer a more reliable diagnosis of it. Studies have indicated that metabolic disorders including glucose, lipid, and cholesterol were tightly associated with PDC, which could be good indicators of it $[40,41]$. In our study, we found lymphocyte counts, the 
number of B cells, and the number of NKT cells changed dramatically between PDC and GC. Therefore, we have tried to establish a diagnostic model based on biochemical parameters and immune cell subsets, which might be more effective for diagnosing and managing of people with PDC. In this combined model, the variables GLU, TG, LDL-C, and lymphocyte counts were finally included, and the area under the curve was 0.878. Interestingly, another group considered PDC as a risk factor of MS and established a diagnostic model including nine related parameters, such as blood sugar, triglycerides, and blood pressure [42]. The area under the curve was 0.865 in their model, which was consistent with our results. In this study, we firstly integrated metabolic and immunity parameters to set an evaluation model distinguishing the ones with PDC from the individuals confused as GC, or other constitution, which might be more suitable for the early-stage screening and health management of individuals with PDC.

In this study, more volunteers should be recruited to improve the representative and accuracy of the diagnostic model, while small sample size might reduce the power of model testing. To remedy this shortcoming, our data were collected by trained staff using standardized instruments according to standardized operating procedures (SOP).

\section{Conclusion}

Phlegm-damp constitution is especially vulnerable to diabetes, MS, and CVD compared with GC. Metabolic inflammation is a key culprit for triggering these metabolicrelated diseases. Here, we firstly integrated metabolic and immunity parameters to establish an objective model for PDC diagnosis and management. It could help screen highrisk patients in the early stages of PDC and offer an evidencebased method for TCM clinical and basis research.

\section{Data Availability}

Data of the figures and tables used to support the findings of this study are included within the article.

\section{Ethical Approval}

This study was approved by the Ethical Review Board of West China Longquan Hospital Sichuan University and Sichuan Academy of Chinese Medicine Sciences. All the participants provided informed consent. The waste generated during the laboratory procedures was disinfected using aseptic techniques and safely disposed.

\section{Conflicts of Interest}

The authors declare that they have no conflicts of interest.

\section{Acknowledgments}

This study was supported by grants from the National Natural Science Foundation of China (no. 81803967).

\section{References}

[1] Q. Wang, "Individualized medicine, health medicine, and constitutional theory in Chinese medicine," Frontiers of Medicine, vol. 6, pp. 1-7, 2012.

[2] J. Wang, Y. Li, C. Ni, H. Zhang, L. Li, and Q. Wang, "Cognition research and constitutional classification in Chinese medicine," The American Journal of Chinese Medicine, vol. 39, no. 4, pp. 651-660, 2011.

[3] W. Qi, "Classification and diagnosis basis of nine basic constitutions in Chinese medicine," Journal of Beijing University Traditional Chinese Medicine, vol. 28, pp. 1-8, 2005.

[4] H. Luo and Q. Wang, "Correlation between TCM constitution and metabolic syndrome: a systematic review and Metaanalysis," Journal of Beijing University of Traditional Chinese Medicine, vol. 39, no. 4, pp. 325-334, 2016.

[5] C. Wang, X. Weiming, and J. Lijie, "Distribution of phlegm and stasis binding pattern in healthy subjects and all stages of coronary heart disease: a multi-centered, cross-sectional study of 11383 cases," Journal of Traditional Chinese Medicine, vol. 62, no. 6, pp. 494-504, 2021.

[6] K. G. M. M. Alberti, R. H. Eckel, S. M. Grundy et al., "Harmonizing the metabolic syndrome," Circulation, vol. 120, no. 16 , pp. 1640-1645, 2009.

[7] M. Aguilar, T. Bhuket, S. Torres, B. Liu, and R. J. Wong, "Prevalence of the metabolic syndrome in the United States, 2003-2012," JAMA, vol. 313, no. 19, pp. 1973-1974, 2015.

[8] A. Ansarimoghaddam, H. A. Adineh, I. Zareban, S Iranpour, A HosseinZadeh, and $\mathrm{F} \mathrm{Kh}$, "Prevalence of metabolic syndrome in Middle-East countries: meta-analysis of cross-sectional studies," Diabetes \& Metabolic Syndrome, vol. 12, no. 2, pp. 195-201, 2018.

[9] S. Kachur, C. J. Lavie, A. de Schutter, R. V Milani, and H. O Ventura, "Obesity and cardiovascular diseases," Minerva Medica, vol. 108, no. 3, pp. 212-228, 2017.

[10] X. N. Tang, D. S. Liebeskind, and A. Towfighi, "The role of diabetes, obesity, and metabolic syndrome in stroke," Seminars in Neurology, vol. 37, no. 3, pp. 267-273, 2017.

[11] T. McLaughlin, S. E. Ackerman, L. Shen, and E. Engleman, "Role of innate and adaptive immunity in obesity-associated metabolic disease," Journal of Clinical Investigation, vol. 127, no. 1, pp. 5-13, 2017.

[12] C. Deng, Y. Xiang, T. Tan et al., "The imbalance of B-lymphocyte subsets in subjects with different glucose tolerance: relationship with metabolic parameter and disease status," Journal of Diabetes Research, vol. 2017, Article ID 5052812, 13 pages, 2017.

[13] S. Torres, E. Fabersani, A. Marquez, and P. Gauffin-Cano, "Adipose tissue inflammation and metabolic syndrome. The proactive role of probiotics," European Journal of Nutrition, vol. 58, no. 1, pp. 27-43, 2019.

[14] H. Qin, J. Jin, and Y. Ren, "Expression and clinical significance of inflammatory factors IL-4, IL-5 and IFN- $\gamma$ in patients with asthma of phlegm-dampness type," Modern Journal of Integrated Traditional Chinese and Western Medicine, vol. 29, no. 32, pp. 3564-3567, 2020.

[15] Q. Zhang, B. Liu, L. Zhao et al., "Venoarterial extracorporeal membrane oxygenation increased immune function of spleen and decreased reactive oxygen species during post-resuscitation," Artificial Organs, vol. 43, no. 4, pp. 377-385, 2019.

[16] Y. Huang, "Correlation of the hyperlipidemia with Chinese medicine constitution in the elderly patients of changfeng community," Shanghai Journal of Medicine, vol. 36, no. 2, pp. 27-29, 2015. 
[17] M. Shi, z. Liu, and M. Xu, "Study on the relationship between traditional Chinese medicine body constitution and chronic disease in the older population in the downtown area of Beijing," Shandong Journal of TCM, vol. 35, no. 1, pp. $28-30+46,2016$.

[18] Q. Wang, C. Jiang, and Y. Gu, "Analyze the constitutions of traditional Chinese medicine in those older subjects with metabolic syndrome in the shanghai community," Modernization of Traditional Chinese Medicine and Materia MateriaWorld Science and Technology, vol. 21, no. 12, pp. 2909-2914, 2019.

[19] D. P. Wang and Q. Wang, "Analysis and expectation of the current research on phlegm-dampness constitution," Chin. J. Tradit. Chin. Med. Pharm, vol. 1, pp. 5-8, 2008.

[20] Q. Wang, J. Dong, and $\mathrm{H}$. Wu, "Study on the molecular characteristic in phlegm-dampness constitution," Engineering Sciences, vol. 7, pp. 100-103, 2008.

[21] D. A. Winer, S. Winer, L. Shen et al., "B cells promote insulin resistance through modulation of T cells and production of pathogenic IgG antibodies," Nature Medicine, vol. 17, no. 5, pp. 610-617, 2011.

[22] L. van Beek, M. A. Lips, A. Visser et al., "Increased systemic and adipose tissue inflammation differentiates obese women with T2DM from obese women with normal glucose tolerance," Metabolism, vol. 63, no. 4, pp. 492-501, 2014.

[23] C. Lorenzo, A. J. Hanley, and S. M. Haffner, "Differential white cell count and incident type 2 diabetes: the Insulin Resistance Atherosclerosis Study," Diabetologia, vol. 57, no. 1, pp. 83-92, 2014.

[24] N. C. Olson, M. F. Doyle, I. H. de Boer et al., "Associations of circulating lymphocyte subpopulations with type 2 diabetes: cross-sectional results from the multi-ethnic study of atherosclerosis (MESA)," PLoS One, vol. 10, no. 10, Article ID e0139962, 2015.

[25] M. Šestan, S. Marinović, I. Kavazović et al., "Virus-induced interferon- $\gamma$ causes insulin resistance in skeletal muscle and derails glycemic control in obesity," Immunity, vol. 49, no. 1, p. 164, 2018.

[26] A. Bendelac, N. Killeen, D. R. Littman, and R. H. Schwartz, “A subset of CD4 + thymocytes selected by MHC class I molecules," Science, vol. 263, no. 5154, pp. 1774-1778, 1994.

[27] A. Bendelac, P. B. Savage, and L. Teyton, "The biology of NKT cells," Annual Review of Immunology, vol. 25, no. 1, pp. 297-336, 2007.

[28] L. Lynch, D. O’Shea, D. C. Winter, J. Geoghegan, D. G. Doherty, and C. O'Farrelly, "Invariant NKT cells and CD1d+cells amass in human omentum and are depleted in patients with cancer and obesity," European Journal of Immunology, vol. 39, no. 7, pp. 1893-1901, 2009.

[29] L. Lynch, M. Nowak, B. Varghese et al., "Adipose tissue invariant NKT cells protect against diet-induced obesity and metabolic disorder through regulatory cytokine production," Immunity, vol. 37, no. 3, pp. 574-587, 2012.

[30] G. S. Getz and C. A. Reardon, "Natural killer T cells in atherosclerosis," Nature Reviews Cardiology, vol. 14, no. 5, pp. 304-314, 2017.

[31] L. Cai, L. Yu, S. Liu et al., "Reconfiguration of NKT cell subset compartment is associated with plaque development in patients with carotid artery stenosis," Inflammation, vol. 40, no. 1, pp. 92-99, 2017.

[32] N. Zhang, M. Zhang, R.-T. Liu et al., "Statins reduce the expressions of Tim-3 on NK cells and NKT cells in atherosclerosis," European Journal of Pharmacology, vol. 821, pp. 49-56, 2018.
[33] J. Wada and H. Makino, "Inflammation and the pathogenesis of diabetic nephropathy," Clinical Science, vol. 124, no. 3, pp. 139-152, 2013.

[34] E. Krauzová, J. Kračmerová, L. Rossmeislová et al., “Acute hyperlipidemia initiates proinflammatory and proatherogenic changes in circulation and adipose tissue in obese women," Atherosclerosis, vol. 250, pp. 151-157, 2016.

[35] E.-G. Hong, H. J. Ko, Y.-R. Cho et al., "Interleukin-10 prevents diet-induced insulin resistance by attenuating macrophage and cytokine response in skeletal muscle," Diabetes, vol. 58, no. 11, pp. 2525-2535, 2009.

[36] H.-J. Kim, T. Higashimori, S.-Y. Park et al., "Differential effects of interleukin- 6 and -10 on skeletal muscle and liver insulin action in vivo," Diabetes, vol. 53, no. 4, pp. 1060-1067, 2004.

[37] B. Hyun, S. Shin, A. Lee et al., "Metformin down-regulates TNF- $\alpha$ secretion via suppression of scavenger receptors in macrophages," Immune Network, vol. 13, no. 4, pp. 123-132, 2013.

[38] A. M. W. Petersen and B. K. Pedersen, "The anti-inflammatory effect of exercise," Journal of Applied Physiology, vol. 98, no. 4, pp. 1154-1162, 2005.

[39] Y. Shen, Z. Duan, and X. Chen, "Association of TCM Constitution with gene polymorphism of interleukin-6,interleukin-10 and interkeukin-10 receptor in patients with Type 2 Diabetes," Journal of Anhui Traditional Chinese Medical College, vol. 38, no. 3, pp. 6-11, 2019.

[40] S. Zhang, S. Zhang, and L. Yun, "Investigation on Chinese medical constitution types of 120 patients with type 2 diabetes mellitus and comparison of glucose metabolism indexes in different constitution," Shanghai Journal of Traditional Chinese Medicine, vol. 54, no. S1, pp. 7-10, 2020.

[41] G.-c. Jing, W.-j. Gu, and M.-r. Zhang, "Study on relationship between constitution of TCM and personality characteristics of patients with type 2 diabetes mellitus," China Journal of Traditional Chinese Medicine and Pharmacy, vol. 32, no. 11, pp. 4946-4948, 2017.

[42] X. Liang, Study on the Screening of Risk Factors the Construction of Diagnostic Models in People with Phlegm-Damp Constitution Prone to Metabolic Syndrome, Beijing University of Chinese Medicine, Beijing, China, 2017. 